\section{Quantitative elastography associated with endoscopic ultrasound for the diagnosis of chronic pancreatitis}

\author{
Julio Iglesias-Garcia, J. Enrique Domínguez-Muñoz, \\ Marga Castiñeira-Alvariño, Maria Luaces-Regueira, \\ Jose Lariño-Noia \\ Endoscopy 2013;45:781-788
}

The diagnosis of chronic pancreatitis (CP) is challenging and as it is difficult to obtain pancreatic biopsy safely, the diagnosis of $\mathrm{CP}$ is based upon the demonstration of morphological and/or functional changes in the pancreas that occurs because of irreversible damage to the pancreatic parenchyma. ${ }^{[1-3]}$ The current cross sectional imaging modalities, although very sensitive and advanced, cannot diagnose early $\mathrm{CP}$ and the functional studies because of associated technical challenges, patient discomfort, and limited availability, are infrequently performed..$^{[4]}$

Because of the ability to use high frequencies and place the transducer in close proximity to the pancreas, endoscopic ultrasound (EUS) provides high resolution images of the pancreas and therefore is considered to be the most sensitive imaging modality for diagnosing pancreatic disorders. It has been shown to demonstrate subtle alterations in the pancreatic parenchymal and ductal structure even before traditional imaging and functional testing demonstrate any abnormality. ${ }^{[4-6]}$ However, there is still no consensus on the EUS criteria for diagnosis of $\mathrm{CP}$ and now we have complicated scoring systems like Rosemont criteria that has multiple parenchymal and ductal EUS features ${ }^{[7]}$ In spite of these criteria the studies have failed to demonstrate any significant improvement in the inter observer variability in diagnosis of $\mathrm{CP}$.

Elastography is a nonhistological method for evaluation of tissue stiffness and EUS elastography has been shown to be an accurate method for differential diagnosis of solid pancreatic tumors. ${ }^{[8]}$ The authors of the current study hypothesized that pancreatic fibrosis in CP is associated with loss of tissue elasticity and this can be measured using quantitative elastography.

The authors prospectively studied 191 patients (mean age 52 years, range 21-85; 103 male and 88 female) who underwent EUS for epigastric pain syndrome $(n=131)$ or were known cases of CP $(n=60)$. All the patients underwent EUS using radial EUS probe (EG 3870 UTK; Pentax Europe GmbH, Hamburg,
Germany) attached to the platform EUB-900 (Hitachi Medical Systems GmbH, Wiesbaden, Germany), which included the elastography module. A stable EUS image for at least $5 \mathrm{~s}$ was required for quantitative elastographic analysis. The region of interest (ROI) was manually selected to include the targeted area of the pancreas as well as surrounding tissues. Area A was the largest possible area of the pancreatic parenchyma and area $B$ was selected as a soft (red) reference area, corresponding to normal surrounding gut wall. The result was defined as the quotient B/A - the strain ratio. Three different elastographic measurements were taken in the head, body, and tail of the pancreas and the final result was the mean value of the three strain ratio values.

The diagnosis of $\mathrm{CP}$ was made using established EUS criteria including the Rosemont criteria. Patients with five or more EUS criteria were diagnosed with $\mathrm{CP}$, whereas it was excluded in cases with $0-2$ criteria. In patients with in conclusive EUS findings, that is, 3 or 4 EUS criteria, a secretin-stimulated Magnetic resonance cholangiopancreatography (s-MRCP) and a contrast-enhanced pancreas magnetic resonance imaging (MRI) with intravenous gadolinium were performed to diagnose $\mathrm{CP}$. If at least one of these findings were present on MRI, the diagnosis of $\mathrm{CP}$ was confirmed: a) ductal changes of $\mathrm{CP}$ after secretin injection according to the Cambridge classification; $b$ ) peak of pancreatic contrast enhancement in the venous or portal phase; and c) gland signal intensity ratio after intravenous gadolinium administration (enhanced/unenhanced) $<1.7$.

According to EUS findings, 99 patients (51.8\%) were considered to have a normal pancreas and $92(48.2 \%)$ were having CP. Seventy patients with $\mathrm{CP}$ had $\geq 5$ EUS criteria, whereas the remaining 22 had 3 or 4 EUS criteria and the diagnosis was supported by MRI/s-MRCP. A homogeneous green elastographic pattern was seen in all patients without $\mathrm{CP}$ and in four patients (4.3\%) with $3(n=3)$ or $4(n=1)$ EUS criteria of CP. A heterogeneous green elastographic pattern was observed in the remaining 88 patients $(95.6 \%)$. The strain ratio was similar in the head, body, and tail of the pancreas, both in patients with a normal pancreas and in those diagnosed with CP. Also, in all three locations, the strain ratio was significantly higher in patients with $\mathrm{CP}$ than in those without the disease. Moreover, the ROC analysis yielded an area under the curve of 0.949 (95\%CI 0.916-0.982) with an accuracy of $91 \%$ at a cut off of 2.25 for strain ratio.

A significantly direct linear correlation was obtained between the strain ratio and the total number of EUS criteria $(\mathrm{r}=0.813 ; P<0.0001)$ as well as between the strain ratio and the number of ductal EUS criteria $(\mathrm{r}=0.779 ; P<0.0001)$ and number of parenchymal EUS criteria $(\mathrm{r}=0.805 ; P<0.0001)$. Patients with equivocal EUS findings ( 3 or 4 EUS criteria) had a significantly higher strain ratio than patients with 0 to 2 EUS criteria (2.41 [95\%CI 2.23-2.60] vs. 1.81 [95\% CI 1.73-1.87], 
respectively). Using the cut-off value for strain ratio of 2.25 , 17 out of the 22 patients $(77.3 \%$ ) with 3 or 4 EUS criteria of $\mathrm{CP}$ had an abnormal pancreas, whereas 5 patients had a strain ratio $<2.25$. Importantly, even in patients with epigastric pain, the strain ratio was significantly higher in those finally diagnosed as CP $(2.68,95 \%$ CI 2.48-2.89) than in whom the disease was excluded (1.80, 95\% CI 1.73-1.87; $P<0.0001)$.

Using Rosemont classification, 99 patients (51.8\%) were considered to have a normal pancreas, 22 (11.5\%) were indeterminate for CP, 45 (23.6\%) were suggestive of $\mathrm{CP}$, and $25(13.1 \%)$ were consistent with CP. The strain ratio was found to be significantly different in the four Rosemont groups $(P<0.001)$. Also, the strain ratio was found to be similar in patients with $\mathrm{CP}$ when compared with $\mathrm{CP}$ of other etiologies as well as in patients with different age groups. The authors concluded that quantitative EUS-elastography associated with EUS morphological assessment of the pancreas appears to be a useful tool for the diagnosis of $\mathrm{CP}$ as specific strain ratios were obtained that showed an excellent accuracy for the diagnosis of CP and thus supporting the more subjective EUS findings.

\section{Commentary}

The diagnosis of early $\mathrm{CP}$ is a challenge and a number of diagnostic modalities such as endoscopic retrograde cholangiopancreatography, magnetic resonance cholangiopancreatography, pancreatic function testing and fecal elastase analysis have been used with varying diagnostic accuracy. The currently available cross sectional imaging modalities can pick up advanced morphological changes of CP only and the diagnostic ability of these modalities to diagnose early and minimal CP is limited. EUS has a unique capability to provide high resolution images of the pancreas and thus has an immense potential for diagnosis of early CP. However, the initial enthusiasm on the role of EUS for diagnosing early $\mathrm{CP}$ has not translated into encouraging results. Poor inter observer agreement, nonspecificity of the most of EUS features of CP and lack of objectivity of the EUS criteria are the major limitations of using EUS for diagnosis of early CP. To overcome some of these limitations of current EUS criteria for diagnosis of $\mathrm{CP}$, Rosemont criteria were developed by leading experts where each EUS criterion was precisely defined so as to have objectivity of the criteria and thus have good inter observer agreement. ${ }^{[6,7]}$ Although, these criteria had refined the past criteria with more objectivity, initial studies have failed to demonstrate any significant improvement in inter observer agreement. ${ }^{[9]}$ Using quantitative EUS elastography for measuring strain ratio to diagnose $\mathrm{CP}$ seems to be interesting and innovative idea and the results of the current study are encouraging. Adding EUS elastography to routine EUS examination that is safe and just adds another 2-3 min to routine EUS procedure, would lend an objective support to the more subjective EUS findings.
However, elastography has some intrinsic limitations like difficulty in controlling tissue compression by the transducer, the motion artifacts secondary to respiratory and heart movements, and the difficulty in excluding nearby structures and intervening cysts and dilated ducts while calculating strain ratios. Also it is recommended that both $\mathrm{A}$ and $\mathrm{B}$ areas should be of equal size but in the current study there was marked inequality in the size of $\mathrm{A}$ and $\mathrm{B}$ areas. ${ }^{[10]}$ In spite of these limitations, the results of this study are encouraging and have shown that EUS elastography has a potential for diagnosis as well as grading the CP. 